University of Nebraska - Lincoln

DigitalCommons@University of Nebraska - Lincoln

7-1995

\title{
Restoration and Reconstruction of AVHRR Images
}

Stephen E. Reichenbach

University of Nebraska - Lincoln, reich@cse.unl.edu

Daniel Kohler

IBM FSCawego, Owego, NY

Dennis Strelow

University of Illinois at Urbana-Champaign, Champaign, IL

Follow this and additional works at: https://digitalcommons.unl.edu/csearticles

Part of the Computer Sciences Commons

Reichenbach, Stephen E.; Kohler, Daniel; and Strelow, Dennis, "Restoration and Reconstruction of AVHRR Images" (1995). CSE Journal Articles. 17.

https://digitalcommons.unl.edu/csearticles/17

This Article is brought to you for free and open access by the Computer Science and Engineering, Department of at DigitalCommons@University of Nebraska - Lincoln. It has been accepted for inclusion in CSE Journal Articles by an authorized administrator of DigitalCommons@University of Nebraska - Lincoln. 


\title{
Restoration and Reconstruction of AVHRR Images
}

\author{
Stephen E. Reichenbach, Daniel E. Koehler, and Dennis W. Strelow
}

\begin{abstract}
This paper describes the design of small convolution kernels for the restoration and reconstruction of Advanced Very High Resolution Radiometer (AVHRR) images. The kernels are small enough to be implemented efficiently by convolution, yet effectively correct degradations and increase apparent resolution. The kernel derivation is based on a comprehensive, end-to-end system model that accounts for scene statistics, image acquisition blur, sampling effects, sensor noise, and postfilter reconstruction. The design maximizes image fidelity subject to explicit constraints on the spatial support and resolution of the kernel. The kernels can be designed with finer resolution than the image to perform partial reconstruction for geometric correction and other remapping operations. Experiments demonstrate that small kernels yield fidelity comparable to optimal unconstrained filters with less computation.
\end{abstract}

\section{INTRODUCTION}

$\mathbf{T}$ HIS paper presents an efficient spatial filter for effectively restoring and reconstructing images from the Advanced Very High Resolution Radiometer (AVHRR). Digital image restoration improves image quality by ameliorating degradations, such as blurring, aliasing, and noise, that are inevitably introduced in the imaging process. Reconstruction defines image values at arbitrary locations in the spatial continuum and is useful for geometric correction, scaling, remapping, and other geometric operations. The filter described here can be efficiently implemented in the spatial domain (or image focalplane) because the spatial support and resolution of the filter is explicitly constrained to a small kernel. Subject to these constraints, the small kernel filter both restores and reconstructs, maximizing image fidelity based on a comprehensive, end-to-end model of the imaging system.

Digital image restoration methods, including the common formulation of the Wiener filter, traditionally have been based on incomplete discrete-input/discrete-output system models that account only for acquisition blur and noise. References [1], [2] formulated the spatially unconstrained Wiener restoration filter based on the continuous-input/discreteimage/continuous-output (CDC) imaging model illustrated in Fig. 1. The CDC Wiener filter maximizes image fidelity

Manuscript received March 29, 1994; revised January 31, 1995. This work was supported in part by the National Science Foundation under contracts CDA-9200654, USE-9152764, and CDA-9022445, in part by the UNL Center for Communication and Information Science, and in part by the National Aeronautics and Space Administration through the Nebraska Space Grant Consortium.

S. E. Reichenbach is with the Department of Computer Science \& Engineering University of Nebraska-Lincoln, Lincoln, NE 68510-0115 USA.

D. E. Koehler is with IBM FSC-Owego, Owego, NY 13827 USA.

D. W. Strelow is with the Department of Computer Sciences University of Illinois at Urbana-Champaign, Champaign, IL 61801 USA.

IEEE Log Number 9412800. (i.e., minimizes mean square error). Filters based on this more comprehensive CDC model outperform traditional filters that are based on discrete/discrete formulations.

Reference [3] described the derivation of optimal spatially constrained restoration kernels based on this CDC model. The derivation of the optimal constrained kernel is consistent with the derivation of the unconstrained CDC Wiener filter, but enforces explicit implementation constraints on the spatial support of the kernel in the design of the filter. In practice, the spatially unconstrained CDC Wiener filter, described in [1], [2], requires significant computation because it is implemented by computing the discrete Fourier transform (DFT) of the image, applying the filter in the frequency domain, and computing the inverse DFT of the result. By constraining the spatial support of the kernel, restoration can be efficiently implemented by convolution in the spatial domain, avoiding the overhead of DFT computations. The computational savings increase with image size, so small kernel convolution is particularly useful with large images such as AVHRR images. Also, small kernel convolution is well suited for parallel implementation because it is local and inherently data-parallel.

This paper presents the derivation of small kernels that not only restore (i.e., correct for systemic degradations), but also reconstruct by interpolating between pixel values. The kernel is constrained to a few elements, but unlike the derivation in [3], the kernel support can be specified to subpixel resolution so that the kernel restores and reconstructs beyond the Nyquist limit of the sampling lattice. This is particular useful for implementing high-fidelity rescaling, remapping, and other geometric operations.

Section II describes the CDC imaging system model and characterizes the model parameters for the AVHRR system based on the work of [4]. Section III presents the derivation of the small kernels for restoration and reconstruction. Section IV presents restoration results for simulated AVHRR images generated from higher-resolution Landsat Multispectral Scanner (MSS) scenes and for an example AVHRR image. The software simulation allows precise analyses of restoration and reconstruction performance; the restoration of the example image demonstrates real-world effectiveness and supports the validity of the simulation. Section $V$ analyzes the computational and storage requirements for small kernel filters and postfilter reconstruction as a function of filter support size and resolution.

\section{END-TO-END AVHRR SYSTEM MODEL}

The end-to-end digital imaging system model in Fig. 1 is the basis for more effective restoration. Unlike many imaging system models, this model accounts for sampling and 


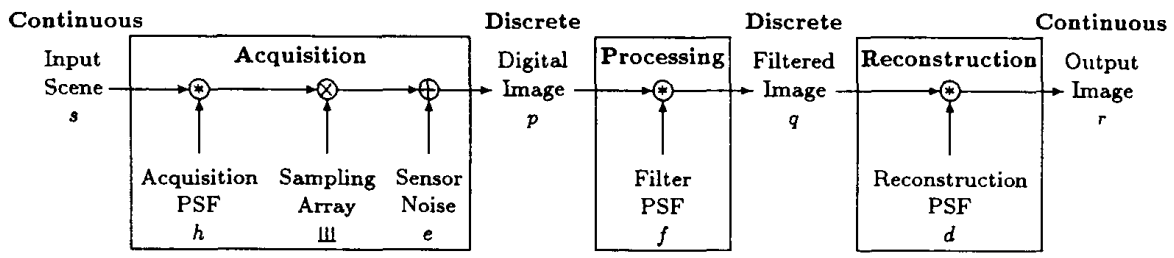

Fig. 1. Mathematical model of the digital imaging process.

\begin{tabular}{|c|c|}
\hline Symbol & Representation \\
\hline \multicolumn{2}{|c|}{ Image Functions } \\
\hline$s$ & Ideal image of scene radiance field \\
\hline$p$ & Digital image \\
\hline$q$ & Filtered digital image \\
\hline$r$ & Reconstructed image \\
\hline \multicolumn{2}{|c|}{ System Functions } \\
\hline$h$ & Acquisition point spread function \\
\hline$e$ & Image noise \\
\hline$f$ & Digital filter \\
\hline$d$ & Post-filter reconstruction function \\
\hline \multicolumn{2}{|c|}{ Function Notation } \\
\hline$\hat{s}$ & Fourier transform (e.g., of $s$ ) \\
\hline$\Phi$, & Autocorrelation (e.g., of $s$ ) \\
\hline$\hat{\Phi}_{s}$ & Power spectrum (e.g., of $s$ ) \\
\hline$\sigma_{s}^{2}$ & Variance (e.g., of $s$ ) \\
\hline \multicolumn{2}{|c|}{ Normalized Coordinates } \\
\hline$x, y$ & Continuous spatial coordinates \\
\hline$m, n$ & Discrete spatial coordinates \\
\hline$u, v$ & Continuous frequency coordinates \\
\hline$\mu, \nu$ & Discrete frequency coordinates \\
\hline \multicolumn{2}{|c|}{ Parameters } \\
\hline$M, N$ & Image dimensions (pixels) \\
\hline$K$ & Kernel size (number of elements) \\
\hline$C$ & Kernel support (location set) \\
\hline$\Delta_{x}, \Delta_{y}$ & Sampling intervals (unnormalized) \\
\hline$\beta$ & Optical blur parameter (unnormalized) \\
\hline$\xi$ & Instantaneous field of view (unnormalized) \\
\hline$\omega$ & TBT filter cutoff (unnormalized) \\
\hline$\kappa$ & TBT phasor angle \\
\hline$T$ & Sample integration scan-distance (unnormalized) \\
\hline$R_{\mathrm{mm}}, R_{\mathrm{n}}$ & Filter resolution (normalized, elements per pixel) \\
\hline$\overline{\mathrm{x}}$ & Scene mean spatial detail (normalized, cycles per pixel) \\
\hline \multicolumn{2}{|c|}{ System Measures } \\
\hline$S^{2}$ & Mean square error \\
\hline$F$ & Fidelity \\
\hline
\end{tabular}

reconstruction, components of digital imaging systems that are frequently ignored but that greatly affect image quality [5]. The model consists of three components: acquisition, processing, and reconstruction. Acquisition is the process of obtaining a digital image from a continuous radiance field. The filter is applied to a digital image to produce an improved digital image. Reconstruction yields a spatially continuous representation from discrete values, e.g., with a display device for viewing or with digital processing for resampling. In a data and information system, the digital image $p$ might be radiometrically corrected satellite data, the filtered image $q$ would be a standard data product available to users, and the output image $r$ would be produced by the user for a specific application. (The symbols used in this paper are listed in Table I).

\section{A. Acquisition}

During acquisition, a digital imaging system blurs, samples, and quantizes. Our characterization of these processes in the AVHRR is from [4]. As in [4], we assume linear, shift-invariant (LSI) blurring; a uniform rectangular sampling lattice; and shift-invariant, signal-independent, additive, white noise. This model is neither complete nor exact, but is an adequate approximation for developing an effective restoration filter.

Mathematically, the spatial-domain acquisition model defines the digital image $p$ at discrete points on the twodimensional sampling lattice identified with integer coordinates $[m . n]$

$$
\begin{gathered}
p[m, n]=\int_{-\infty}^{\infty} \int_{-\infty}^{\infty} h(m-x, n-y) s(x, y) d x d y \\
+e[m, n]
\end{gathered}
$$

where the ideal image of the scene radiance field $s$ is convolved with the acquisition point spread function (PSF) $h$ and $e$ is the additive random noise. For notational convenience, the spatial coordinates $(x, y)$ are normalized to the sampling interval in each dimension and brightness values are expressed on a gray-level equivalent scale. The corresponding spatialfrequency-domain acquisition model is

$$
\begin{gathered}
\hat{p}(u, v)=\sum_{\mu=-\infty}^{\infty} \sum_{\nu=-\infty}^{\infty} \hat{h}(u-\mu, v-\nu) \hat{s}(u-\mu, v-\nu) \\
+\hat{e}(u, v)
\end{gathered}
$$

where $u$ and $v$ are spatial frequencies (normalized to the sampling frequency), $\hat{p}$ is the Fourier transform or spatialfrequency spectrum of the image, $\hat{h}$ is the acquisition transfer function, $\hat{s}$ is spatial frequency spectrum of the scene, and $\hat{e}$ is the spatial frequency spectrum of the noise (which is periodic with the sampling frequency). Sampling causes the folding of the components of the spatial frequency spectrum and the resulting image transform $\hat{p}$ is periodic with period equal to the normalized sampling frequency, 1.0 in each dimension.

The AVHRR system is a whisk-broom scanner, where the field is projected onto a row of detectors in a sweeping motion (along-scan) orthogonal to the path of the satellite (alongtrack). The optical transfer function (OTF) is the Fourier transform of the LSI PSF and is used to characterize acquisition blurring. In the AVHRR, the OTF differs in the along-scan and along-track dimensions. The OTF model in the along-scan dimension has four components: optics, detector, electronic filter, and sample integration. The OTF model in the along-track (or cross-scan) dimension has only the optical and detector components. The two-dimensional OTF model is the separable product of the along-scan and along-track functions. 
TABLE II

AVHRR System Model Parameters (5-BANd Models) [4]

\begin{tabular}{|c|r|r|}
\hline Band & $\begin{array}{c}\text { Blur } \beta \\
\text { (meters) }\end{array}$ & $\begin{array}{c}\text { IFOV } \xi \\
\text { (meters) }\end{array}$ \\
\hline 1 & 266.72 & 1195.36 \\
2 & 276.20 & 1191.19 \\
3 & 383.42 & 1141.21 \\
4 & 362.10 & 1182.86 \\
5 & 322.11 & 1095.40 \\
\hline
\end{tabular}

The optics are modeled as

$$
\hat{h}_{o}(u, v)=\exp \left(-\left(u \beta / \Delta_{x}\right)^{2}\right) \exp \left(-\left(v \beta / \Delta_{y}\right)^{2}\right)
$$

where $\Delta_{x}$ and $\Delta_{y}$ are the along-scan and along-track pixel sampling intervals and $\beta$ is the diameter of the blur circle approximating the optics PSF. The ground-projected dimensions for the blur circle diameter $\beta$ for the five channel AVHRR systems (including NOAA 7,9, and 11) are given in Table II. In these systems, the ground-projected dimension of $\Delta_{x}$ is $791.35 \mathrm{~m}$ and the ground-projected dimension of $\Delta_{y}$ is equal to the instantaneous field of view (IFOV) $\xi$ in Table II. In the AVHRR, blurring caused by the optics is relatively small compared to the blurring caused by other components such as the detector.

The detector is modeled as a rectangular function in the spatial domain and as a sinc function in the spatial-frequency domain

$$
\hat{h}_{d}(u, v)=\operatorname{sinc}\left(u \xi / \Delta_{x}\right) \operatorname{sinc}\left(v \xi / \Delta_{y}\right)
$$

where $\operatorname{sinc}(x)=\sin (\pi x) /(\pi x)$.

The electronic filter is a low-pass filter that reduces highfrequency noise with blurring in the along-scan dimension. The electronic filter model is a fourth-order transitional Butterworth-Thompson (TBT) filter

$$
\begin{gathered}
\hat{h}_{e}(u, v)=\left(\left(u \omega / \Delta_{x}\right)^{4}-i \kappa_{3}\left(u \omega / \Delta_{x}\right)^{3}-\kappa_{2}\left(u \omega / \Delta_{x}\right)^{2}\right. \\
\left.+i \kappa_{1}\left(u \omega / \Delta_{x}\right)+1\right)^{-1}
\end{gathered}
$$

where $\omega$ controls the filter cutoff and the values $\kappa_{1}, \kappa_{2}$, and $\kappa_{3}$ are functions of a single parameter $\kappa$ that provides a linear variation in the phasorv angle [6]. With $\kappa=0$, the pole position is that of the Butterworth filter; with $\kappa=1$, the pole position is that of the Thompson filter; with $\kappa=0.5$, the pole is the geometric mean between the two. For the five-band AVHRR, $\omega=1502.3 \mathrm{~m}$ and $\kappa=0.8$, so the filter is nearer the Thompson filter, with more blurring and less overshoot. This yields $\kappa_{3}=3.0256, \kappa_{2}=4.2033$, and $\kappa_{1}=3.0943$.

Sample integration is modeled as a one-dimensional rectangular function in the spatial domain and as a sinc function in the spatial-frequency domain

$$
\hat{h}_{s}(u, v)=\operatorname{sinc}\left(u \tau / \Delta_{x}\right)
$$

where $\tau$ is the scan distance during the sample integration is $94.2 \mathrm{~m}$ (ground-projected) for the five-band AVHRR systems [4]. Because the distance involved in sample integration is small relative to the detector size and pixel spacing, scanner blur is comparatively insignificant.

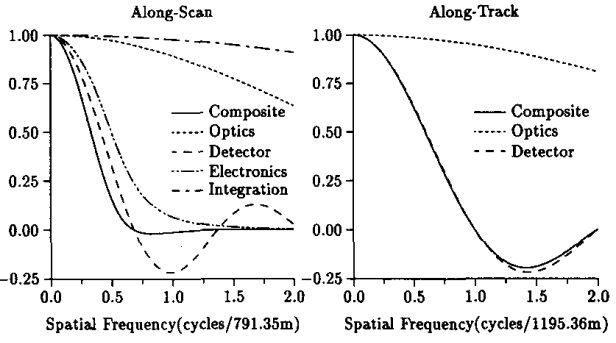

Fig. 2. AVHRR acquisition transfer functions ( 5 band model, channel 1).
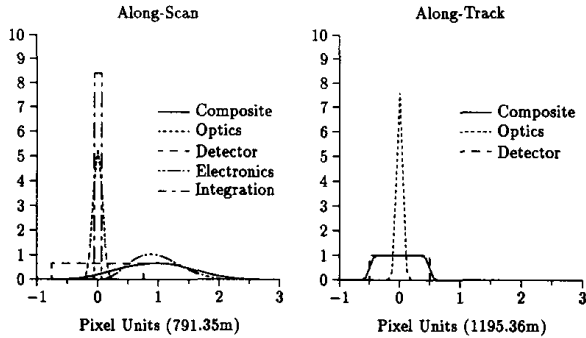

Fig. 3. AVHRR acquisition point spread functions ( 5 band model, channel 1).

The composite, two-dimensional OTF is

$$
\hat{h}(u, v)=\hat{h}_{o}(u, v) \hat{h}_{d}(u, v) \hat{h}_{e}(u, v) \hat{h}_{s}(u, v) .
$$

The composite acquisition transfer function of Band 1 of the 5-band AVHRR model and the individual components are graphed in Fig. 2. Spatial frequencies are normalized to the unit sampling frequency in each dimension. Note that although the optical component is radially symmetric, its blurring effect relative to the inter-pixel spacing is larger in the along-scan dimension than in the along-track dimension. In both dimensions, the detector is the most significant source of blurring at frequencies within the Nyquist limit $(|u|<0.5$ and $|v|<0.5$ ). The electronics transfer function and sample integration are present only in the along-scan dimension. Only the magnitude of the complex-valued electronics transfer function is shown. (The other component transfer functions are real only, so the overall acquisition transfer function has the same imaginary component as the electronics transfer function.)

The phase shift of the electronics is more effectively illustrated in Fig. 3, which graphs the composite and component PSF's. In both the along-scan and along-track dimensions, the detector blurring dominates the optical blurring. As is evident in Fig. 3, the electronics introduces a shift of about one pixel in the along-scan dimension. In Section IV, the digital image is shifted left one pixel before processing to largely compensate for this shift introduced by the TBT filter.

With respect to restoration, it is clear from examining the composite transfer functions in Fig. 2, that in the along-scan dimension there is significant blurring, with little response beyond the Nyquist limit. In the along-track dimension, there is significant response beyond the Nyquist limit, with a response of about 0.6 at frequency 0.5 , and a significant response 
beyond frequency 1.0 where there is phase reversal. Restoration in the along-scan dimension is principally limited by the system noise with little aliasing. In the along-track dimension, there is less blurring to correct, but the image is degraded by both noise and aliasing of high-frequency spectral components.

All digital imaging systems suffer from noise. Sources include random photon flux, circuit noise, and quantization. Here, we use an additive white noise model characterized by the expected variance of the noise. The signal-to-noise ratio (SNR) is defined as the square root of the ratio of the expected signal variance to the expected noise variance

$$
\mathrm{SNR}=\frac{\sigma_{s}}{\sigma_{e}} .
$$

Previous measurements of AVHRR noise yield estimates for $\sigma_{e}$ of between 0.4 and 1.5 bits on a 10 bit scale [4].

\section{B. Digital Filter}

The digital filter $f$ takes the input digital image $p$ and produces a filtered digital image $q$. If the digital filter has the same resolution as the input image, the filtered image also will have the same resolution as the unprocessed digital image. (With reference a digital image or filter, we use the term resolution here to mean the resolution limit imposed by the sampling lattice, i.e., number of elements per unit interval. Other factors also determine the apparent resolution of an end-to-end digital imaging system.) However, the digital filter need not have the same resolution as the input image and this allows for reconstructing images with finer spatial resolution than the input image during restoration. For example, if the filter is designed with twice the resolution in each dimension as the image (i.e., two elements per pixel in each dimension), then the filtered image will have twice the resolution in each dimension as the input image. For simplicity, we will consider only filters with resolution that is an integer multiple of the resolution of the image $p$. (Actually, this approach allows arbitrary resolution using a higher resolution and constraining the spatial support of the filter as described in Section III.)

If we define the filter $f$ on a lattice that has $R_{m} \times R_{n}$ elements per pixel (indicating the coordinates of filter elements with integer indices divided by filter resolution), then the filtered image $q$ is

$$
\begin{aligned}
& q\left[\frac{m}{R_{m}}, \frac{n}{R_{n}}\right] \\
& \quad=\sum_{m^{\prime}=-\infty}^{\infty} \sum_{n^{\prime}=-\infty}^{\infty} f\left[\frac{m}{R_{m}}-m^{\prime}, \frac{n}{R_{m}}-n^{\prime}\right] p\left[m^{\prime}, n^{\prime}\right]
\end{aligned}
$$

Of course as a practical matter, only a finite image $p$ is available for processing and the spatial support of a digital filter must be constrained. To address these issues and to facilitate the use of the DFT, it is common to assume the scene and hence the image are periodic with period equal to the image size and to constrain the filter support to the size of the image (or smaller). This means the filtering operation is one of circular convolution which can be implemented using the DFT. The frequency-domain equation corresponding to (9) is

$$
\hat{q}(u, v)=\hat{f}(u, v) \hat{p}(u, v)
$$

With the filter resolution limited to $R_{m} \times R_{n}$ elements per pixel, the filter transfer function $\hat{f}$ is periodic with period $R_{m} \times R_{n}$ times the sampling frequency. With the filter resolution equal to a multiple of the image resolution, the resolution of the filtered image $q$ is the same as that of the filter, and its transform $\hat{q}$ is also periodic with period $R_{m} \times R_{n}$ times the sampling frequency. With a periodic image and constrained filter, the transforms in (10) are discrete as well as periodic, and can be computed with the DFT.

If the spatial support of the filter is the size of the image, the filter is global, and the only practical implementation for moderately sized and large images is to use a fast Fourier transform (FFT) to compute the image spatial frequency spectrum $\hat{p}$, multiply by the filter transfer function [as in (10)], and compute the inverse Fast Fourier transform of the product. However, for a $M \times N$ image, the FFT is $O(M N \lg (M N))$, and even for moderately sized images the FFT requires significant computation. Filters with small spatial support e.g., a local filter may be implemented more efficiently with convolution in the spatial domain, as in (9). Convolution for a $M \times N$ image and filter with $K$ elements is $O(K M N)$. If $K$ is small, then convolution requires less computation; if $K$ is large, then frequency-domain computation requires less computation. For a specific computer, one can define the break-even point for the support size $K$ as a function of image size. As an approximate general guideline for workstation-class computers, convolution is more efficient if $K<2.5 \lg (M N)$ [7]. Not only is small kernel convolution efficient, but parallel implementation is straightforward.

\section{Reconstruction}

Reconstruction produces a continuous image from a digital image. A display monitor produces a spot for each pixel value, effectively blurring the discrete pixel values to form a continuous image. Display system designs seek uniformity in homogeneous regions without excessive blurring of sharp transitions [5]. A Gaussian PSF is commonly used to model the display spots of video monitors. Traditional digital resampling operations also implicitly convolve the digital image with a continuous reconstruction function even though the result is computed only at the resampled points. Nearest-neighbor (or sample-and-hold), bilinear, and cubic convolution [8] are common digital reconstruction methods that use continuous reconstruction PSF's. LSI reconstruction with reconstruction PSF $d$ is

$r(x, y)=\sum_{m=-\infty}^{\infty} \sum_{n=-\infty}^{\infty} d\left(x-\frac{m}{R_{m}}, y-\frac{n}{R_{m}}\right) q\left[\frac{m}{R_{m}}, \frac{n}{R_{m}}\right]$.

The corresponding frequency-domain equation is

$$
\hat{r}(u, v)=\hat{d}(u, v) \hat{q}(u, v)
$$

where $\hat{d}$ is the reconstruction transfer function.

\section{Scene Model}

The autocorrelation of the scene is required for the filter derivation in Section III. The scene autocorrelation is modeled 
as

$$
\Phi_{s}(x, y)=\sigma_{s}^{2} \exp \left(-\sqrt{x^{2}+y^{2}} / \bar{x}\right)
$$

where the parameter $\bar{x}$ is the mean spatial detail (or correlation length) of the object or scene (in normalized pixel units). This function is the non-separable autocorrelation of a circularly symmetric Markov process and is widely used for modeling scene autocorrelation [9], [10]. The mean spatial detail $\vec{x}$ parameterizes the "correlation length" of the autocorrelation function. The corresponding scene power spectrum model is

$$
\hat{\Phi}_{s}(u, v)=\frac{2 \pi \bar{x}^{2} \sigma_{s}^{2}}{\left(1+4 \pi^{2} \bar{x}^{2}\left(u^{2}+v^{2}\right)\right)^{3 / 2}} .
$$

For the results presented in Section IV, the brightness variance $\sigma_{s}^{2}$ is 1024 times the noise variance $\sigma_{e}^{2}$, so as defined in (8) SNR $=32$. Various values for the scene mean spatial detail $\bar{x}$ of AVHRR scenes are considered in Section IV, but filters derived for $\bar{x}=1$ work fairly well for a range of spatial detail.

\section{Derivation OF the SMall Kernel}

The small restoration kernel minimizes the expected mean square difference between the ideal image of the scene and the reconstructed image, subject to constraints on spatial support and resolution. With reference to Fig. 1, the expected mean square error is

$$
\begin{aligned}
S^{2} & =E\left\{\int_{-\infty}^{\infty} \int_{-\infty}^{\infty}|s(x, y)-r(x, y)|^{2} d x d y\right\} \\
& =E\left\{\int_{-\infty}^{\infty} \int_{-\infty}^{\infty}|\hat{s}(u, v)-\hat{r}(u, v)|^{2} d u d v\right\} .
\end{aligned}
$$

Reference [11] used the expected mean square error to define image fidelity

$$
F=1-\frac{S^{2}}{\sigma_{s}^{2}}
$$

where $\sigma_{s}^{2}$ is the expected (ensemble average) variance of the ideal image of the scene radiance field

$$
\begin{aligned}
\sigma_{s}^{2} & =E\left\{\int_{-\infty}^{\infty} \int_{-\infty}^{\infty}|s(x, y)|^{2} d x d y\right\} \\
& =E\left\{\int_{-\infty}^{\infty} \int_{-\infty}^{\infty}|\hat{s}(u, v)|^{2} d u d v\right\}
\end{aligned}
$$

For notational convenience, we assume a zero-mean scene process in these equations; in practice, the image mean can be adjusted during filtering. Fidelity is bounded by 1 , with equality if the output image is identical to the scene radiance field. Mean-square-error metrics such as fidelity are intuitive, facilitate mathematical analyses, and may correlate better with performance in automated recognition and classification, but they do not directly correspond to human assessments of visual quality. While there is no general acknowledgment of a better objective definition of visual quality, it is possible to introduce ad hoc adjustments for better subjective visual quality [12]. The issues surrounding the utility of restoration in automated recognition and classification are considered in Section VI.
The $C D C$ Wiener filter $f_{w}$ has no constraints on the spatial size or limit on the resolution [1]

$$
\hat{f}_{w}(u, v)=\frac{\hat{\Phi}_{s, p}(u, v)}{\hat{\Phi}_{p}(u, v)} \frac{\hat{d}^{*}(u, v)}{|d(u, v)|^{2}}
$$

(the “ $*$ " superscript denotes complex conjugation) where $\hat{\Phi}_{s, p}$ is the cross power spectrum of the scene and image

$$
\begin{aligned}
\hat{\Phi}_{s, p}(u, v) & =E\left\{\hat{s}(u, v) \hat{p}^{*}(u, v)\right\} \\
& =\hat{\Phi}_{s}(u, v) \hat{h}^{*}(u, v) .
\end{aligned}
$$

$\hat{\Phi}_{p}$ is the image power spectrum

$$
\begin{aligned}
\hat{\Phi}_{p}(u, v) & =E\left\{|\hat{p}(u, v)|^{2}\right\} \\
= & \sum_{\mu=-\infty}^{\infty} \sum_{\nu=-\infty}^{\infty} \hat{\Phi}_{s}(u-\mu, v-\nu)|\hat{h}(u-\mu, v-\nu)|^{2} \\
& +\hat{\Phi}_{e}(u, v)
\end{aligned}
$$

and $\hat{\Phi}_{s}$ and $\hat{\Phi}_{e}$ are the power spectra of the scene and noise, respectively.

On a digital computer, the filter resolution must be limited. Let the filter be limited to $R_{m} \times R_{n}$ elements/pixel, with postfilter reconstruction function $d$ (either for display or subsequent digital resampling): Then, the optimal limited-resolution filter $f_{l}$ is [2]

$$
\hat{f}_{l}(u, v)=\frac{\hat{b}(u, v)}{\hat{a}(u, v)}
$$

where

$$
\begin{array}{r}
\hat{a}(u, v)=\sum_{\mu=-\infty}^{\infty} \sum_{\nu=-\infty}^{\infty} \hat{\Phi}_{p}\left(u-\mu R_{m}, v-\nu R_{n}\right) \\
\quad \times\left|\hat{d}\left(u-\mu R_{m}, v-\nu R_{n}\right)\right|^{2} \\
\hat{b}(u, v)=\sum_{\mu=-\infty}^{\infty} \sum_{\nu=-\infty}^{\infty} \hat{\Phi}_{s, p}\left(u-\mu R_{m}, v-\nu R_{n}\right) \\
\times \hat{d}^{*}\left(u-\mu R_{m}, v-\nu R_{n}\right) .
\end{array}
$$

The limited-resolution filter transfer function is periodic with $R_{m} \times R_{n}$ cycles/pixel. If $R>1$, then this filter yields a digital image with increased resolution. Further reconstruction is required for continuous output or for arbitrary resampling, but with adequate resolution, the constrained filter can effectively implement a significant part of the reconstruction operation.

We also constrain the spatial support of the filter to a small set of locations $C$ on the lattice defined by the limiting resolution of the filter. The elements not in the support set $C$ cannot be used in the filter; i.e., the filter is zero at these locations

$$
f_{c}\left[\frac{m}{R_{m}}, \frac{n}{R_{n}}\right]=0 \quad \text { if }\left[\frac{m}{R_{m}}, \frac{n}{R_{n}}\right] \notin C .
$$

The integers $R_{m}$ and $R_{n}$ in the denominators of the coordinates allow the filter to have finer resolution than the image with $R_{m} \times R_{n}$ elements per pixel interval. 
With resolution and support constrained, the optimal small kernel filter $f_{\mathrm{c}}$ is defined by the linear equations

$\sum_{\left[\frac{m^{\prime}}{R_{m}}, \frac{n^{\prime}}{R_{m}}\right] \in C} a\left[\frac{m-m^{\prime}}{R_{m}}, \frac{n-n^{\prime}}{R_{n}}\right] f_{c}\left[\frac{m^{\prime}}{R_{m}}, \frac{n^{\prime}}{R_{n}}\right]=b\left[\frac{m}{R_{m}}, \frac{n}{R_{n}}\right]$

for $\left[m / R_{m}, n / R_{n}\right] \in C$ where $a$ and $b$ are the inverse transforms of $\hat{a}$ and $\hat{b}$ [(in (22) and (23)], respectively and the number of unknowns and number of equations are equal to the number of elements in the filter constraint set. The optimal values for the constrained kernel are defined by the solution for $f_{c}$ in (25).

As a practical matter, we compute $\hat{a}$ and $\hat{b}$ using finite discrete functions for the scene power spectra $\hat{\Phi}_{s}$, acquisition transfer function $\hat{h}$, and reconstruction transfer function $\hat{d}$. Then, $a$ and $b$ can be computed using the inverse FFT algorithm. In doing this, the frequency cutoff that is used must be sufficiently large that relatively little error is introduced by truncating the spatial frequency spectra. For example, in Section 4, the small kernels for the AVHRR are derived using a cutoff of 16 times the sampling frequency.

The expected mean square error (15) for the CDC Wiener filter $f_{u}$ is

$$
S_{w}^{2}=\int_{-\infty}^{\infty} \int_{-\infty}^{\infty} \hat{\Phi}_{s}(u, v)-\left|\hat{\Phi}_{s, p}(u, v)\right|^{2} / \hat{\Phi}_{p}(u, v) d u d v .
$$

Therefore, the fidelity (16) for this filter is

$$
F_{w}=\frac{1}{\sigma_{s}^{2}} \int_{-\infty}^{\infty} \int_{-\infty}^{\infty}\left|\hat{\Phi}_{s, p}(u, v)\right|^{2} / \hat{\Phi}_{p}(u, v) d u d v
$$

For any filter $f$, the expected mean square error is

$$
\begin{aligned}
S^{2}=S_{w}^{2}+\int_{-\infty}^{\infty} \int_{-\infty}^{\infty} & \hat{\Phi}_{p}(u, v)|d(u, v)|^{2} \\
& \times\left|\hat{f}(u, v)-\hat{f}_{w}(u, v)\right|^{2}
\end{aligned}
$$

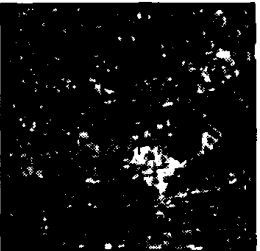

(a)

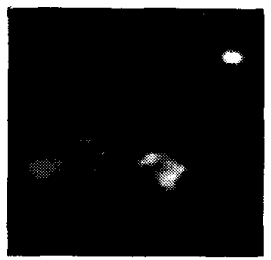

(d)

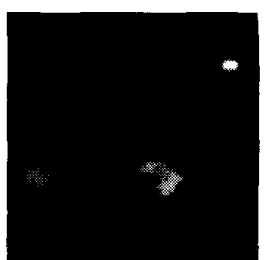

(b)

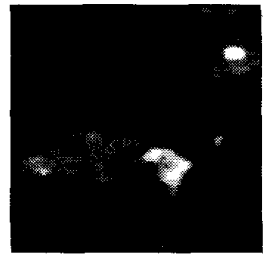

(e)

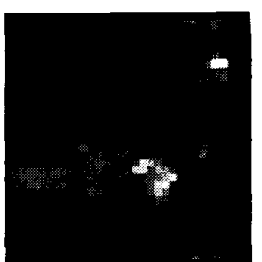

(c)

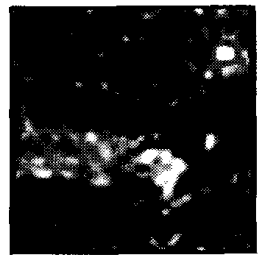

(f)

Fig. 4. AVHRR system simulation with four conventional-reconstruction methods and with the CDC Wiener filter. (a) MSS scene, (b) Gaussian, (c) nearest-neighbor, (d) Bilinear, (e) cubic convention, (f) CDC Wiener filter $f_{u}$.

\section{RESULTS}

This section presents results for both simulated and actual AVHRR images. In Section IV-A, software simulation of the AVHRR system allows assessment of overall system performance with precision, control, and flexibility that is not possible with real images [13]. Section IV-B presents results for an actual AVHRR image. The similarity of the simulated image to the actual image provides a basis for confidence in the simulation results.

\section{A. Simulated AVHRR Images}

The AVHRR simulation presented here uses a $512 \times 512$ MSS image as the input scene $s$. To simulate AVHRR acquisition, the MSS scene is blurred, sampled, and corrupted with additive noise as described in Section II. The sampling ratio in this simulation is 16:1 (16 MSS pixels to 1 AVHRR pixel), so the simulated AVHRR image $p$ is $32 \times 32$. This ratio is slightly higher than the actual MSS:AVHRR sampling ratio, but is close enough for the purpose of the experiments presented here.

Restoration with the CDC Wiener filter yields an image that is more similar to the scene than does display reconstruction or conventional interpolation. Fig. 4 pictures the results of the end-to-end AVHRR system simulation. Fig. 4(a) is a MSS Band 2 scene from central Nebraska, including the city of Kearney. MSS Band 2 is used as the scene for simulated imaging of AVHRR Band 1. An actual AVHRR Band 1 image of this region taken within a day of this MSS image is presented in Section IV-B. Fig. 4(b) is the image after simulated AVHRR acquisition, no digital filter, and reconstruction with a simulated display monitor using a Gaussian spot with root-mean-square (RMS) radius 0.5 pixels. Fig. $4(\mathrm{c})$ is the image after simulated AVHRR acquisition, no digital filter, and reconstruction with nearest-neighbor interpolation. This image most clearly illustrates the pixel resolution. Fig. 4 (d) is 


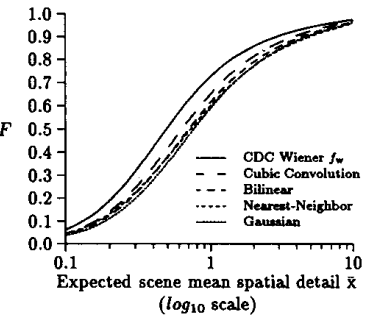

(a)

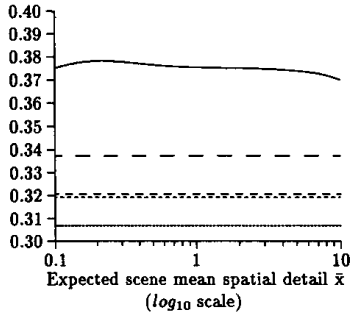

(b)
Fig. 5. Fidelity for four conventional reconstruction methods and for the CDC Wiener filter $f_{w}$. (a) Expected fidelity, (b) example fidelity.

the image after simulated AVHRR acquisition, no-digital filter, and reconstruction with bilinear interpolation. Fig. 4(e) is the image after simulated AVHRR acquisition, no digital filter, and reconstruction with cubic convolution. Fig. $4(f)$ is the image after simulated AVHRR acquisition and digital restoration and reconstruction with the CDC Wiener filter $f_{w}$.

A great deal of detail in the original MSS scene is lost in the imaging process and cannot be restored, but the CDC Wiener filter yields a sharper image with greater detail (e.g., along the high contrast edge running west from the center of the city) than do the conventional reconstruction functions. Although we present images to illustrate visually the differences, we are primarily interested in the accuracy of the quantitative measures that are especially relevant in automated processing. With increasing volumes of data, automated processing will be increasingly necessary and important.

The expected fidelity with the CDC Wiener filter is significantly better than with display reconstruction or conventional interpolation. Fig. 5(a) graphs the expected fidelity for AVHRR images with CDC Wiener restoration, cubic convolution, bilinear interpolation, nearest-neighbor interpolation, and Gaussian reconstruction as a function of scene mean spatial detail $\bar{x}$ [as determined by (29)]. Among the simple reconstruction methods, cubic convolution yields the highest expected fidelity and Gaussian reconstruction the lowest. This graph also illustrates that the spatial detail of the scene is the important determinant of image fidelity; for all reconstruction methods, if the scene detail is small relative to the sampling frequency then the fidelity is low and if the scene detail is large relative to the sampling frequency then the fidelity is high.

The predominant effect of the actual mean spatial detail on actual fidelity can be seen in Fig. 5(b), which graphs the fidelity for the example image in Fig. 4 . This is a relatively detailed scene with a small mean spatial detail. Therefore, the digital image is significantly degraded by the blurring and undersampling of the simulated AVHRR acquisition and the fidelity is low regardless of the method of restoration or reconstruction. Note that the performance of the CDC Wiener filter depends on the mean-spatial-detail parameter used in defining the filter. In Fig. 5(b), the abscissa indicates the presumed mean spatial detail presumed in the design of the CDC Wiener filter. (Only the CDC Wiener filter is a function of the presumed scene mean spatial detail. Bilinear interpolation and the other reconstruction methods
TABLE III

Fidelity for Four Conventional Reconstruction Methods AND FOR THE CDC WiENER FILER $f_{w}$, DESIGNED FOR $\bar{x}=1$

\begin{tabular}{|l|c|c|}
\hline & $\begin{array}{c}\text { Expected } \\
\text { Fidelity } \\
\overline{\mathrm{x}}=1\end{array}$ & $\begin{array}{c}\text { Example } \\
\text { Fidelity } \\
\overline{\mathrm{x}} \approx 0.25\end{array}$ \\
\hline CDC Wiener $f_{\mathrm{w}}$ & 0.725 & 0.375 \\
\hline Cubic Convolution & 0.650 & 0.337 \\
\hline Bilinear & 0.614 & 0.321 \\
\hline Nearest-Neighbor & 0.599 & 0.319 \\
\hline Gaussian & 0.589 & 0.307 \\
\hline
\end{tabular}

TABLE IV

FiDELITY FOR THE LIMTTED-RESOLUTION (SPATIALly UNCONSTRAINED) Wiener Filter $f_{l}$ with Four Postfilter Reconstruction Methods

\begin{tabular}{|l|c|c|c|}
\hline \multicolumn{1}{|c|}{ Post-Filter } & \multicolumn{3}{|c|}{ Filter Resolution } \\
Reconstruction & $R_{\mathrm{m}}=R_{\mathrm{n}}=1 \quad R_{\mathrm{m}}=R_{\mathrm{n}}=2$ & $R_{\mathrm{m}}=R_{\mathrm{n}}=4$ \\
\hline Cubic Convolution & 0.718 & 0.725 & 0.725 \\
\hline Bilinear & $\mathbf{0 . 7 1 1}$ & $\mathbf{0 . 7 2 4}$ & $\mathbf{0 . 7 2 5}$ \\
\hline Nearest-Neighbor & $\mathbf{0 . 6 2 1}$ & $\mathbf{0 . 6 9 2}$ & 0.718 \\
\hline Gaussian & 0.717 & 0.724 & 0.725 \\
\hline
\end{tabular}

A. Expected fidelity $(\overline{\mathrm{x}}=1)$.

\begin{tabular}{|l|c|c|c|}
\hline \multicolumn{1}{|c|}{ Post-Filter } & \multicolumn{3}{|c|}{ Filter Resolution } \\
\multicolumn{1}{c|}{ Reconstruction } & $R_{\mathrm{m}}=R_{\mathrm{a}}=1$ & $R_{\mathrm{m}}=R_{\mathrm{n}}=2$ & $R_{\mathrm{m}}=R_{\mathrm{n}}=4$ \\
\hline Cubic Convolution & $\mathbf{0 . 3 7 3}$ & 0.375 & 0.375 \\
\hline Bilinear & $\mathbf{0 . 3 7 1}$ & $\mathbf{0 . 3 7 4}$ & $\mathbf{0 . 3 7 5}$ \\
\hline Nearest-Neighbor & $\mathbf{0 . 3 3 1}$ & $\mathbf{0 . 3 5 6}$ & $\mathbf{0 . 3 7 1}$ \\
\hline Gaussian & $\mathbf{0 . 3 7 4}$ & $\mathbf{0 . 3 7 4}$ & 0.375 \\
\hline
\end{tabular}

B. Example fidelity $(\bar{x} \approx 0.25)$.

are not premised on any presumptions about the scene statistics.)

As expected, the CDC Wiener filter yields significantly better fidelity than display reconstruction or conventional interpolation. It can be inferred both from the mean spatial detail where the fidelity for the CDC Wiener filter peaks and from the fidelity at the peak that (13) best models the scene in Fig. 4 with mean spatial detail $\bar{x}$ approximately 0.2 to 0.3 pixels. This graph also indicates that the CDC Wiener filter is relatively robust with respect to the mean spatial detail; for example, the fidelity for the filter based on $\bar{x}=1$ works nearly as well as filters based on smaller estimates of $\bar{x}$. This is good because it means that the method is fairly insensitive to errors in estimating the scene mean spatial detail and suggests that a global average can be used relatively effectively for non-homogeneous scenes.

The CDC Wiener filter used for Fig. 4(f) is premised on a mean spatial detail $\bar{x}=1$. This is a slight mismatch, because, as just described, the actual mean spatial detail is 0.2 to 0.3 pixels. However, this is perhaps better indicative of a typical result where the mean spatial detail is mis-estimated or the global estimate is used for shift-invariant processing. The numeric values of the expected fidelity and example fidelity (with $\bar{x}=1$ presumed) are given in Table III. (The example fidelity is computed using the actual mean square difference between the scene and processed images.) The limited resolution and constrained filters presented later in this paper are similarly robust with respect to mean spatial detail $\bar{x}$, so for the subsequent results in this paper only the numeric values for $\bar{x}=1$ are given.

Table IV presents expected and example fidelity for the optimal limited-resolution filter $f_{l}$ with four different methods 
TABLE V

Fidelity for the SMall Kernel Filter $f_{c}$ WITH Cubic-Convoluttion Postfilter Reconstruction. The Cubic INTERPOLATOR IS DEFINED FOR THE RESOLUTION OF THE FILTER

\begin{tabular}{|c|c|c|c|c|}
\hline Post-Filter & Size & \multicolumn{3}{|c|}{ Filter Resolution } \\
\hline Reconstruction & (Pixels) & $R_{\mathrm{m}}=R_{\mathrm{n}}=1$ & $R_{\mathrm{m}}=R_{\mathrm{n}}=2$ & $R_{\mathrm{m}}=R_{\mathrm{n}}=4$ \\
\hline \multirow{3}{*}{ Cubic Convolution } & $3 \times 3$ & 0.708 & 0.707 & 0.706 \\
\hline & $5 \times 5$ & 0.716 & 0.718 & 0.719 \\
\hline & $7 \times 7$ & 0.717 & 0.722 & 0.722 \\
\hline \multicolumn{5}{|c|}{ A. Expected fidelity $(\ddot{x}=1)$. } \\
\hline \multirow{2}{*}{$\begin{array}{c}\text { Post-Filter } \\
\text { Reconstruction }\end{array}$} & Size & \multicolumn{3}{|c|}{ Filter Resolution } \\
\hline & (Pixels) & $R_{\mathrm{m}}=R_{\mathrm{n}}=1$ & $R_{\mathrm{m}}=R_{\mathrm{n}}=2$ & $R_{\mathrm{m}}=R_{\mathrm{n}}=4$ \\
\hline \multirow{3}{*}{ Cubic Convolution } & $3 \times 3$ & 0.367 & 0.365 & 0.366 \\
\hline & $5 \times 5$ & 0.372 & 0.372 & 0.372 \\
\hline & $7 \times 7$ & 0.373 & 0.374 & 0.374 \\
\hline
\end{tabular}

B. Example fidelity $(\bar{x} \approx 0.25)$.

of postfilter reconstruction. Even for resolution limited to the pixel resolution, $R_{m}=R_{n}=1$, the optimal limitedresolution filter with cubic convolution, bilinear interpolation, or Gaussian postfilter reconstruction yields fidelity nearly as good as the CDC Wiener filter which has unlimited resolution. With nearest-neighbor postfilter reconstruction, however, the fidelity of the optimal limited-resolution filter is considerably less than for the CDC Wiener filter and is even less than for simple cubic convolution with no restoration. The fidelity with nearest-neighbor interpolation is lower because nearestneighbor interpolation does a poor job of estimating values between pixels. The frequency domain explanation of this is instructive. The transfer function (or spatial frequency response) at normalized spatial frequencies beyond the Nyquist limit \pm 0.5 is larger for nearest-neighbor interpolation than for the other reconstruction transfer functions. Generally, the system should attenuate components at these frequencies, but if the filter is limited to be periodic with the sampling frequency it cannot attenuate these components without attenuating components at frequencies less than the Nyquist limit. For filter resolution equal to twice the pixel resolution, $R_{m}=R_{n}=2$, the fidelity with nearest-neighbor postfilter reconstruction method is significantly improved, although it is still not as good as with the other postfilter reconstruction methods. For filter resolution equal to four times the pixel resolution, $R_{m}=R_{n}=4$, the fidelity for the optimal limitedresolution filter with nearest-neighbor postfilter reconstruction is nearly as good as for the CDC Wiener filter and the optimal limited-resolution filter. The other postfilter reconstruction methods yields fidelity nearly identical to that for the CDC Wiener filter.

Table $\mathrm{V}$ presents expected and example fidelity for the small kernel filter with three different small regions of spatial support, $3 \times 3$ pixels, $5 \times 5$ pixels, and $7 \times 7$ pixels. (Note these are the sizes of the filter support in pixel units. The number of elements in the kernel is a function of both size and filter resolution.) Only the results for cubic-convolution postfilter reconstruction are given. The results for bilinear, nearest-neighbor, and Gaussian postfilter reconstruction are ordered relatively as in Table IV, with Gaussian and bilinear postfilter reconstruction doing not quite as well as cubicconvolution and with nearest-neighbor reconstruction yielding the lowest fidelity. Some example small kernels designed for

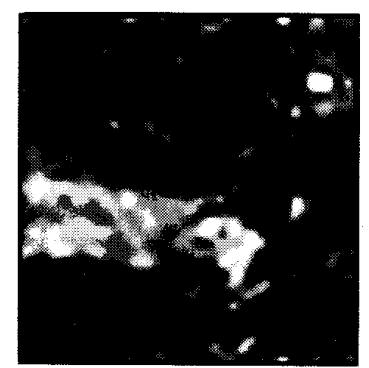

Fig. 6. Result image for the $R_{m}=R_{n}=1,3 \times 3$ small kernel filter $f_{c}$ with cubic-convolution postfilter reconstruction at the filter-resolution.

AVHRR image restoration and reconstruction are given in the Appendix.

Even the smallest kernels with the lowest resolution perform nearly as well as the CDC Wiener filter which has unlimited resolution and unconstrained size. Fidelity improves with increasing spatial support and the $7 \times 7$ kernels are large enough to nearly match the performance of the CDC Wiener filter. The tradeoffs between fidelity and computational expense associated with kernel size, resolution, and postfilter processing are considered in Section V.

Fig. 6 shows the restoration of the simulated image in Fig. 4, for the $R_{m}=R_{n}=1,3 \times 3$ small kernel with cubic-convolution postfilter reconstruction. This image is quite similar to the image produced by the CDC Wiener filter shown in Fig. 4(f), but the raster pattern and artifacts of subsequent reconstruction are more evident. As will be seen, increasing filter resolution decreases these artifacts.

When we began getting the results from these experiments, we were surprised that in some cases, increasing the resolution of small kernels caused a slight reduction in fidelity. For example, in Table $\mathrm{V}$, the expected fidelity for the $3 \times 3$ small kernel with cubic convolution postfilter reconstruction is 0.708 for $R_{m}=R_{n}=1,0.707$ for $R_{m}=R_{n}=2$, and 0.706 for $R_{m}=R_{n}=4$. The same decrease is observed in the example image, with fidelity 0.367 for $R_{m}=R_{n}=1$ and 0.365 for $R_{m}=R_{n}=2$. The decrease in actual fidelity might have been attributable to the inevitable inaccuracy of the scene power spectrum model, but the decrease in expected fidelity forced us to look for the real explanation.

For the results in Table $\mathrm{V}$, the postfilter reconstruction functions are designed for the resolution of the filtered image. For example, for resolution $R_{m}=R_{n}=1$ the cubic postfilter interpolation PSF has a $4 \times 4$ pixels spatial extent, for resolution $R_{m}=R_{n}=2$ the cubic convolution PSF is $2 \times 2$ pixels, and for resolution $R_{m}=R_{n}=4$ the cubic convolution PSF is $1 \times 1$ pixels. Therefore, different postfilter reconstruction functions are used at each of the three filter resolutions. Most reconstruction transfer functions roll-off at about \pm 0.5 times the resolution for which they are designed. This means that for filters with resolution $R_{m}=R_{n}=1$ there is a rolloff in the postfilter reconstruction transfer function at about \pm 0.5 , for resolution $R_{m}=R_{n}=2$ there is a roll-off in the reconstruction transfer function at about \pm 1 , and for resolution $R_{m}=R_{n}=4$ there is a roll-off in the reconstruction transfer 
TABLE VI

Fidelity for the SMall KeRnel Filter $f_{c}$ WITH Cubic-Convolution POSTFILter Reconstruction. The Cubic INTERPOLATOR IS DEFINED FOR THE PIXEL. RESOLUTION

\begin{tabular}{|c|c|c|c|}
\hline $\begin{array}{c}\text { Post-Filter } \\
\text { Reconstruction }\end{array}$ & Size & \multicolumn{2}{|c|}{ Filter Resolution } \\
(Pixels) & $R_{\mathrm{m}}=R_{\mathrm{n}}=2$ & $\boldsymbol{R}_{\mathrm{m}}=\boldsymbol{R}_{\mathrm{m}}=4$ \\
\hline & $3 \times 3$ & 0.718 & 0.719 \\
Cubic Convolution & $5 \times 5$ & $\mathbf{0 . 7 2 2}$ & $\mathbf{0 . 7 2 3}$ \\
& $7 \times 7$ & 0.724 & 0.724 \\
\hline
\end{tabular}

A. Expected fidelity $(\tilde{\mathbf{x}}=1)$.

\begin{tabular}{|c|c|c|c|}
\hline \multirow{2}{*}{$\begin{array}{c}\text { Post-Filter } \\
\text { Reconstruction }\end{array}$} & \multirow{2}{*}{$\begin{array}{c}\text { Size } \\
\text { (Pixels) }\end{array}$} & \multicolumn{2}{|c|}{ Filter Resolution } \\
\hline & & $R_{\mathrm{m}}=R_{\mathrm{n}}=2$ & $R_{m}=R_{n}=4$ \\
\hline \multirow{3}{*}{ Cubic Convolution } & $3 \times 3$ & 0.372 & 0.373 \\
\hline & $5 \times 5$ & 0.374 & 0.374 \\
\hline & $7 \times 7$ & 0.375 & 0.375 \\
\hline
\end{tabular}

B. Example fidelity $(\bar{x} \approx 0.25)$.

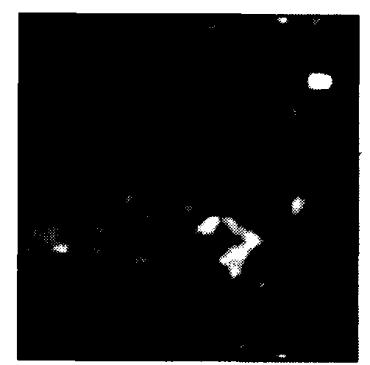

Fig. 7. Result image for the $R_{m}=R_{n}=2,5 \times 5$ small kernel filter $f$ with cubic-convolution postfilter reconstruction at the filter-resolution.

function at about \pm 2 . Typically, it is desirable to attenuate spatial-frequency components above the Nyquist limit of the sampling lattice, i.e., beyond \pm 0.5 . Hence, with increasing filter resolution, the postfilter reconstruction function does less attenuation of high-frequency components, leaving the task to the restoration filter. The larger small-kernels are large enough to effectively accomplish the blurring, but the $3 \times 3$ kernels are not.

This led us to consider using reconstruction functions designed for the pixel resolution, $R_{m}=R_{n}=1$, regardless of the filter resolution. In this case, the cubic postfilter interpolation PSF has spatial extent of $4 \times 4$ pixels regardless of the filter resolution. The results of these experiments are presented in Table VI. The fidelity of the higher-resolution small kernels is better with postfilter reconstruction functions designed for the pixel resolution than with postfilter reconstruction functions designed for the filter resolution. As described above, this is because the postfilter reconstruction functions designed for the pixel resolution perform more of the desired attenuation of spatial-frequency components above the Nyquist limit of the sampling lattice. However, this is not without cost; the postfilter reconstruction functions designed for the pixel resolution require more computation than the smaller postfilter reconstruction functions designed for the filter resolution. The computational costs are examined in Section V.

Fig. 7 shows the restoration for a $R_{m}=R_{n}=2$ resolution small-kernel with cubic-convolution postfilter reconstruction defined for the image resolution. The image is very similar to the image produced by the CDC Wiener filter shown in Fig. 4(f).

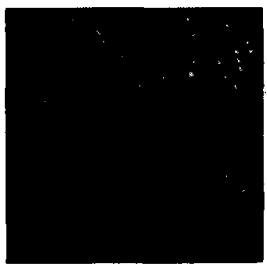

(a)

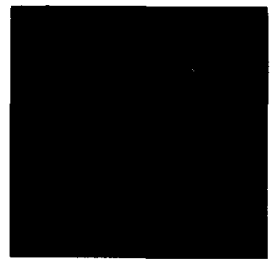

(d)

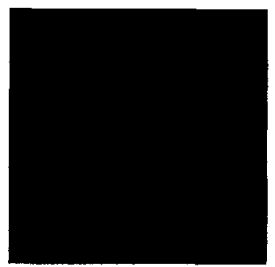

(b)

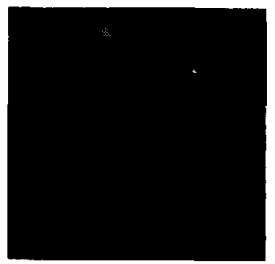

(e)

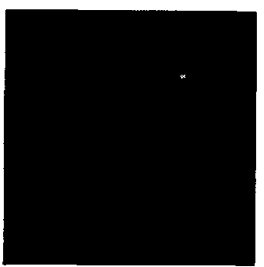

(c)

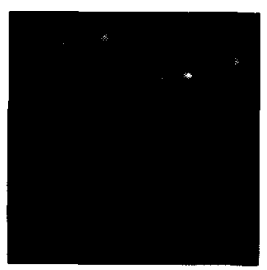

(f)
Fig. 8. AVHRR system simulation with Gaussian reconstruction, with the CDC Wiener filter, and with three small kernel filters. (a) MSS scene, (b) Gaussian, (c) CDC Wiener $f_{w}$, (d) $f_{c}$ with $R_{m}=R_{n}=1$ and $3 \times 3$, (e) $f_{c}$ with $R_{m}=R_{n}=2$ and $5 \times 5$, (f) $f_{c}$ with $R_{m}=R_{n}=4$ and $7 \times 7$.

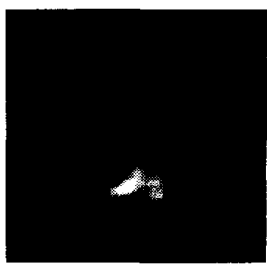

(a)

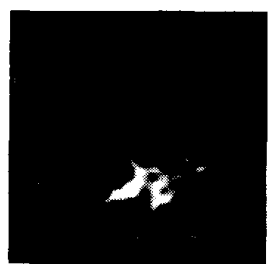

(b)

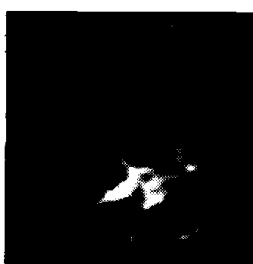

(c)
Fig. 9. Actual AVHRR image with Gaussian reconstruction, with the CDC Wiener filter, and with the small kernel filter. (a) Gaussian, (b) CDC Wiener $f_{w}$, (c) small kernel $f_{c}$.

Fig. 8 illustrates another AVHRR simulation. Fig. 8(a) is a MSS Band 1 scene from another location along the Platte River valley in Nebraska, but with different spatial character than the image in Fig. 4. Fig. 8(b) is the Gaussian spot reconstruction of the simulated AVHRR image and Fig. 8(c) is the CDC Wiener restoration. Fig. 8(d)-(f) are small kernel restorations with cubic-convolution postfilter reconstruction, Fig. 8(d) for $R_{m}=R_{n}=1$ and $3 \times 3$, Fig. 8(e) for $R_{m}=R_{n}=2$ and $5 \times 5$, and Fig. 8(f) for $R_{m}=R_{n}=4$ and $7 \times 7$. The fidelity for these images are 0.652 for Gaussian reconstruction; for $0.687 \mathrm{CDC}$ Wiener restoration; and $0.683,0.687$, and 0.687 for the three small kernel restorations. Visually, one can see that the scene in Fig. 8 is not as detailed as the scene in Fig. 4. The fidelity indicates that (13) best models the scene in Fig. 4 with mean spatial detail $\bar{x} \approx 1$ pixel.

\section{B. Actual AVHRR Images}

The AVHRR image presented in Fig. 9 is of the same general vicinity and was taken within a day of the MSS scene in Fig. 4. Fig. 9(a) is a $32 \times 32$ AVHRR image reconstructed to $512 \times 512$ with a Gaussian PSF with RMS radius 0.5 pixels (and no restoration). The character of this image is very similar to that of the corresponding simulated image 
TABLE VII

Relative Computational and Storage Costs

\begin{tabular}{|c|c|c|c|}
\hline & \multicolumn{3}{|c|}{ Filter Resolution $\left(R_{m}=R_{n}\right)$} \\
\hline & 1 & & \\
\hline Computation for $3 \times 3$ Filter & $9 M N$ & $49 M N$ & $169 M N$ \\
\hline Computation for $5 \times 5$ Filter & $25 M N$ & $121 M N$ & $441 M N$ \\
\hline Computation for $7 \times 7$ Filter & $49 M N$ & $225 M N$ & $841 M N$ \\
\hline Storage for Filtered Image & $M N$ & $4 M N$ & $16 M N$ \\
\hline $\begin{array}{l}\text { Computation for Nearest Neighbor } \\
\text { at Filter Resolution* }\end{array}$ & $M N$ & $M N$ & $M N$ \\
\hline $\begin{array}{l}\text { Computation for Bilinear at Filter } \\
\text { Resolution }\end{array}$ & $4 M N$ & $4 M N$ & $4 M N$ \\
\hline $\begin{array}{l}\text { Computation for Cubic Convolution } \\
\text { (or Gaussian) at Filter Resolution }{ }^{*}\end{array}$ & $8 M N$ & $8 M N$ & $8 M N$ \\
\hline $\begin{array}{l}\text { Computation for Nearest Neighbor } \\
\text { at Image Resolution* }\end{array}$ & $M N$ & $4 M N$ & $8 M N$ \\
\hline $\begin{array}{l}\text { Computation for Bilinear at Image } \\
\text { Resolution* }\end{array}$ & $4 M N$ & $8 M N$ & $16 M N$ \\
\hline $\begin{array}{l}\text { Computation for Cubic Convolution } \\
\text { (or Gaussian) at Image Resolution" }\end{array}$ & $8 M N$ & $16 M N$ & $32 M N$ \\
\hline
\end{tabular}

in Fig. 4(b). Note, however, that the two images are not geometrically registered and the scales of the two images differ slightly. Fig. 9(b) is restored and reconstructed with the CDC Wiener filter and Fig. 9(c) is restored with a $R_{m}=$ $R_{n}=1,3 \times 3$ small kernel with cubic-convolution postfilter reconstruction. The two restored images are similar to one another and to the restoration results for the corresponding simulated images in Figs. 4(f) and 6. The similarity of the simulated image to the actual AVHRR image indicates that the AVHRR simulation is a reasonable approximation and that the experimental assessments and the conclusions presented in Section IV-A are meaningful.

\section{COMPUTATIONAL CONSIDERATIONS}

There are many possible small kernels. One naturally asks: "Which small kernel should I use?" Unfortunately, there is no single answer to this question. Generally, higher fidelity requires more computation, so one must either answer the question: "What fidelity is required?" and then choose the filter that delivers the desired fidelity and requires least computation, or answer the question: "How much computation can be expended?" and then choose the filter that maximizes fidelity within the computational limit. This task may be complicated by the fact that computation may be required first for filtering and then for postfilter reconstruction. One or both of these steps may be subject to processing constraints related to the hardware used for each step.

Table VII illustrates the relative costs for filtering computation, filtered image storage, and postfilter processing. This chart, with those in Section IV-A, makes some choices clear. For example, the $R_{m}=R_{n}=4,3 \times 3$ small kernel is more expensive and yields lower fidelity than the $R_{m}=R_{n}=2$, $5 \times 5$ small kernel. Other cases require case-specific costbenefit analysis of the tradeoffs between fidelity and cost.

\section{CONCLUSION}

The small kernels derived in this paper efficiently and effectively restore AVHRR images. The approach is based on a comprehensive end-to-end system model, provides explicit control over computation via constraints on spatial support
TABLE VIII

EXAMPLE $R_{m}=R_{n}=1,3 \times 3$ SMALl KeRnels $f_{c}$ to BE USED WITH Bilinear Postfilter Reconstruction and with Convolution Postfilter Reconstruction

\begin{tabular}{|r|rrr|rrr|}
\hline & \multicolumn{3}{|c|}{ Bilinear } & \multicolumn{3}{|c|}{ Cubic Convolution } \\
\hline & 0.1565 & -0.4407 & 0.1254 & 0.0889 & -0.2436 & 0.0693 \\
Band 1 & -0.7992 & 2.6958 & -0.6383 & -0.5574 & 2.0908 & -0.4238 \\
& 0.1565 & -0.4407 & 0.1254 & 0.0889 & -0.2436 & 0.0693 \\
\hline & 0.1564 & -0.4407 & 0.1253 & 0.0889 & -0.2437 & 0.0692 \\
Band 2 & -0.7979 & 2.6939 & -0.6370 & -0.5564 & 2.0892 & -0.4227 \\
& 0.1564 & -0.4407 & 0.1253 & 0.0889 & -0.2437 & 0.0692 \\
\hline & 0.1560 & -0.4437 & 0.1246 & 0.0889 & -0.2469 & 0.0690 \\
Band 3 & -0.7850 & 2.6763 & -0.6240 & -0.5453 & 2.0742 & -0.4115 \\
& 0.1560 & -0.4437 & 0.1246 & 0.0889 & -0.2469 & 0.0690 \\
\hline & 0.1590 & -0.4479 & 0.1276 & 0.0907 & -0.2490 & 0.0707 \\
Band 4 & -0.8042 & 2.7098 & -0.6426 & -0.5609 & 2.1014 & -0.4267 \\
& 0.1590 & -0.4479 & 0.1276 & 0.0907 & -0.2490 & 0.0707 \\
\hline & 0.1487 & -0.4291 & 0.1178 & 0.0843 & -0.2375 & 0.0648 \\
Band 5 & -0.7521 & 2.6127 & -0.5926 & -0.5191 & 2.0236 & -0.3867 \\
& 0.1487 & -0.4291 & 0.1178 & 0.0843 & -0.2375 & 0.0648 \\
\hline
\end{tabular}

and resolution, and maximizes image fidelity subject to the spatial constraints. The kernels can be designed with greater resolution than the image to perform partial reconstruction for geometric correction and other remapping operations. Small kernel convolution can be implemented in parallel for real-time restoration and reconstruction. Simulation results demonstrate that the small kernels can be nearly as effective as more computationally expensive unconstrained filters.

The future utility of restoration of remote sensing data is more significant for automated processing than for visual processing. The Earth Observing System (EOS) program [14] will generate more data than humans can or will examine directly. This data is being generated primarily for automated processing. Reference [15] argues that the problems of restoring for subsequent visual or automated examination are different problems that probably have different solutions. They demonstrate that in controlled TM simulations, restoration can significantly reduce classification errors. Still, the problem of defining a restoration method tailored for classification accuracy is a difficult problem. For example, [15] reports better classification with what they call partial restoration in which the filter does not correct for detector blurring. This reflects the fact that the restored value is used as a measure for the neighborhood rather than as point measure. Such partial restoration is a special case of the technique that [2] call characteristic restoration and can be implemented with a small kernel [16]. As these results indicate, more research into the use of restoration to enhance automated processing is needed.

\section{APPENDIX}

EXAMPLE SMALL KeRNELS

Table VIII illustrates the $R_{m}=R_{n}=1,3 \times 3$ small kernels to be used with bilinear postfilter reconstruction and with cubic-convolution postfilter reconstruction.

- These filters are to be applied after the image is shifted one pixel to the left.The left shift largely compensates for the shift introduced during acquisition (Fig. 3). The filters are index reversed during convolution (9). A brightness offset is added during filtering so that the brightness mean is unchanged. 
- The small kernels designed to be used with bilinear postfilter reconstruction perform greater sharpening than the small kernels to be used with cubic-convolution postfilter reconstruction. This is because cubic convolution is a "sharper" reconstruction function than bilinear interpolation.

- The small kernel filter is nearly the same for all bands. This is because the AVHRR acquisition function is nearly the same for all bands.

- The small kernel filters sharpen more along-scan than along-track. This is because the AVHRR acquisition system introduces more blurring along-scan than along-track. (Fig. 2)

\section{REFERENCES}

[1] F. O. Huck, C. L. Fales, N. Halyo, R. W. Samms, and K. Stacy, "Image gathering and processing: Information and fidelity," J. Opt. Soc. Amer. $A$, vol. 2 , no. 10 , pp. $1644-1666,1985$.

[2] C. L. Fales, F. O. Huck, J. A. McCormick, and S. K. Park, "Wiener restoration of sampled image data: End-to-end analysis," J. Opt. Soc. Amer. A, vol. 5, no. 3, pp. $300-314,1988$.

[3] S. E. Reichenbach and S. K. Park, "Small convolution kernels for highfidelity image restoration," IEEE Trans. Signal Processing, vol. 39, pp. 2263-2274, Oct. 1991.

[4] R. Schowengerdt and W. M. Li, "Modify U. of Arizona restoration software module system (AVHRR image deconvolution/resampling capability)," Final Rep., USGS Contract No. PO 060206-86, Feb. 1987.

[5] W. F. Schreiber, Fundamentals of Electronic Imaging Systems: Some Aspects of Image Processing. New York: Springer-Verlag, 1986.

[6] Y. Peless and T. Murakami, "Analysis and synthesis of transitional Butterworth-Thompson filters and bandpass amplfiers," RCA Rev., vol. 18 , pp. $60-94,1957$.

[7] S. E. Riechenbach, "Small-kernel image restoration," Ph.D. dissertation, College of William and Mary, Williamsburg, VA, July 1989.

[8] S. K. Park and R. A. Schowengerdt, "Image reconstruction by parametric cubic convolution," Computer Vision, Graphics Image Processing, vol. 23, pp. 258-272, 1983.

[9] Y. Itakura, T. Suteo, and T. Takagi, "Statistical properties of the background noise for the atmospheric windows in the intermediate infrared region," Infrared Phys., vol. 14, pp. 17-29, 1974.
[10] K. Ben-Yosef, N. Wilner, S. Simhony, and G. Feigin, "Measurement and analysis of 2-D infrared natural background," Appl. Opt., vol. 24, no. 14, pp. 2109-2113, 1985.

[11] E. H. Linfoot, "Transmission factors and optical design," J. Opt. Soc. Amer., vol. 46, no. 9, pp. 740-752, 1956.

[12] J. A. McCormick, R. Alter-Gartenberg, and F. O. Huck, "Image gathering and restoration: Information and visual quality," J. Opt. Soc. Amer. $A$, vol. 6, no. 7, pp. 987-1005, 1989.

[13] S. E. Reichenbach, S. K. Park, R. Alter-Gartenberg and Z. Rahman, "Artificial scenes and simulaed imaging," Proc. SPIE, pp. 422-433, 1991.

[14] G. Asrar and D. J. Dokken, Eds., EOS Reference Handbook, NASA 1993.

[15] H.-H. P. Wu and R. A. Schowengerdt, "Improved estimation of fraction images using partial image restoration," IEEE Trans. Geosci. Remote Sensing, vol. 31, pp. 771-778, July 1993.

[16] S. E. Reichenbach, S. K. Park, and R. Alter-Gartenberg, "Optimal, small kernels for edge detection," in Proc. Int. Conf. Pattern Recognition, IEEE Computer Society, 1990, vol. II, pp. 57-63.

Stephen E. Reichenbach received the B.S. degree from the University of Nebraska, Lincoln, the M.S. degree from Washington University, St. Louis, MO, and the Ph.D. degree from College of William and Mary, Williamsburg, VA, in 1976, 1985, and 1989, respectively.

$\mathrm{He}$ is an Assistant Professor in the Computer Science and Engineering Department at the University of Nebraskaa, Lincoln. His research interests are in digital image processing, including image restoration, enhancement, compression, and automated analysis. His research focuses on resource constraints in end-to-end imaging system performance, especially multispectral remote sensing systems and infrared imagers. He holds an ASEE Fellowship at the NASA Goddard Space Flight Center working in the Landsat 7 Project Science Office.

Daniel E. Koehler, photograph and biography not available at the time of publication.

Dennis W. Strelow, photograph and biography not available at the time of publication. 